Revista de Psicología Vol. 33 (1), 2015 (ISSN 0254-9247)

\title{
El consenso estratégico como predictor de resultados de equipos de trabajo
}

\author{
Katia Puente-Palacios ${ }^{1}$, Tatiana Moreira ${ }^{2}$, Tamara Puente ${ }^{3}$ y Naianne Lira ${ }^{4}$ \\ Universidad de Brasilia-Brasil ${ }^{124}$ y Pontificia Universidad Católica del Ecuador ${ }^{3}$
}

El consenso estratégico en equipos de trabajo es un proceso grupal relativo a la comprensión compartida entre los integrantes, sobre las estrategias definidas para alcanzar las metas de trabajo. El estudio realizado buscó verificar la capacidad de predicción del consenso estratégico con relación al desempeño. Los datos fueron recogidos de docentes y coordinadores de 70 planteles educativos ecuatorianos, a partir de los que se construyó el modelo de predicción. Las respuestas individuales fueron agregadas por plantel para obtener puntajes grupales. Los resultados mostraron que el consenso estratégico predice aproximadamente $6 \%$ de los resultados del desempeño del equipo evaluado por el coordinador. Se concluye que son necesarios más estudios para entender mejor el papel del consenso en equipos de trabajo. Palabras clave: consenso estratégico, desempeño de equipos, predicción del desempeño

\section{Strategic consensus as a predictor for team performance}

Strategic consensus in work teams is a group process related to the shared comprehension among team members of the strategies defined to attain work goals. This study aimed to verify the predictive power of strategic consensus in relation to team performance.

$1 \quad \mathrm{PhD}$ en Psicología por la Universidad de Brasilia (Brasil) y docente del posgrado (Maestría y Doctorado) en Psicología Social, del Trabajo y de las Organizaciones de la Universidad de Brasilia. Dirección postal: Universidad de Brasilia, Instituto de Psicología, Campus Darcy Ribeiro. Brasilia-DF. CEP-70910-900. Contacto: kep.palacios@gmail.com

$2 \mathrm{MsC}$ en Psicología Social, del Trabajo y de las Organizaciones por la Universidad de Brasilia. Profesora temporaria de la Universidad de Brasilia, Departamento de Psicología Social y del Trabajo. Dirección postal: Universidad de Brasilia, Instituto de Psicología, Campus Darcy Ribeiro. Brasilia-DF. CEP-70910-900. Contacto: tatiana.moreira@gmail.com

$3 \mathrm{MsC}$ en Ciencias de la Educación por la Pontificia Universidad Católica del Ecuador. Es docente de la misma universidad, en la Facultad de Ciencias de la Educación. Dirección postal: Av. 12 de Octubre 1076 y Roca. Contacto: tamarapuen@yahoo.es

4 Graduada en el curso de Psicología. Diploma obtenido en la Universidad de Brasilia. Miembro del grupo de investigación Dequi - que estudia el desempeño de equipos de trabajo-, en el Departamento de Psicología Social y del Trabajo de esa universidad. Dirección postal: Universidad de Brasilia, Instituto de Psicología, Campus Darcy Ribeiro. Brasilia-DF. CEP70910-900. Contacto: naiannecmlira@gmail.com 
The prediction model was constructed based on data collected from teachers and coordinators of 70 educational institutions in Ecuador. The individual data were aggregated per institution to obtain group level scores. The results indicate that strategic consensus predicts about $6 \%$ of the team performance as rated by the coordinator. We concluded that more studies are required to gain a better understanding of the role of strategic consensus in work teams.

Keywords: strategic consensus; team performance; performance prediction 
El acuerdo establecido entre los miembros del equipo acerca de las estrategias de trabajo adoptadas para alcanzar los resultados esperados es un fenómeno conocido en el ámbito de la psicología organizacional como consenso estratégico, cuya manifestación es considerada generalmente como el anticipó de un desempeńo eficaz. Autores de este campo destacan que, a pesar del tiempo transcurrido desde su proposición, el tema mantiene el interés de estudiosos del área, y demanda la continuidad de investigaciones empíricas (Walter, Kellermanns, Floyd, Veiga \& Matherne, 2013). La existencia de la relación entre el consenso estratégico y los resultados alcanzados por la organización ha sido demostrada por diversos autores e incluye un estudio de tipo metaanálisis (Kellermanns, Walter, Floyd, Lechner \& Shaw, 2011). De la misma manera, estudios realizados anteriormente por Kellermanns, Walter, Lechner y Floyd (2005), y Mohammed y Ringseis (2001), sugieren que el papel del consenso puede depender de la presencia de otras variables contextuales o mantener relaciones curvilíneas. Por lo tanto, la realización de investigaciones sobre esta temática se muestra como una contribución relevante y necesaria, puesto que aún hay dudas sobre el papel que desempeña el consenso en el ámbito de los equipos.

La importancia de constatar la asociación entre consenso estratégico y desempeño es más obvia si se recuerda que cada día es mayor el número de organizaciones diversas que se organizan en torno a equipos. Su implementación se justifica por la creciente complejidad del mundo de trabajo que genera un sinnúmero de desafíos, muchos de los cuales difícilmente pueden ser enfrentados a partir de estructuras basadas en el desempeño individual. Los equipos deben desarrollar la capacidad de actuar de manera coordinada, sincronizada, permitiendo la manifestación del potencial de sus miembros. Por lo tanto, esa modalidad de trabajo presupone la existencia de acuerdos entre los integrantes en cuanto a las tareas a ser realizadas y a la forma más eficaz de alcanzar las metas. 
La constatación de la presencia de equipos como unidades de trabajo en las empresas no es nueva, pues su prominencia ya era referida desde los ańos noventa por Cohen y Bailey (1997) y continúa siendo vista hoy en día. Así, los gestores y estudiosos del área exigen una mayor comprensión de los fenómenos que subyacen al funcionamiento de los equipos, pues ellos son parte del cotidiano de trabajo del mundo organizacional, y su funcionamiento y desempeńo pueden alterar resultados del trabajo individual, grupal y de la organización como un todo.

Sobre el funcionamiento de los equipos, debe ser destacado que uno de los aspectos que ha despertado interés refiere a los procesos o transformaciones acaecidas durante la experiencia de trabajo compartida por sus integrantes. Chan (1998), al abordar las transformaciones que suceden en situaciones de trabajo conjunto, relata que pueden adoptar diversos matices y resultar en el surgimiento de nuevos fenómenos que no existen en el nivel individual, pero que son característicos de agrupamientos, como los equipos. Esto significa que al estar en contacto con los otros miembros, nuevas formas de interpretar los fenómenos, nuevas competencias, nuevas actitudes y, en general, nuevos atributos pueden surgir. Ellos son propios del equipo y no son características del individuo aislado, sino que se concretizan y aparecen a partir de las relaciones interpersonales que tienen como trasfondo el trabajo compartido. Esos son los fenómenos que nacen por el proceso conocido bajo el nombre de emersión (algo que emerge).

Abordando específicamente el proceso de surgimiento o emersión del consenso estratégico, Moreira y Puente-Palacios (2014) lo describen como una cognición (conocimiento o saber sobre algo) que se origina en los individuos, miembros del equipo, a partir de relaciones de interacción interpersonal generan algo nuevo, que es compartido por los miembros, y da lugar a un fenómeno eminentemente colectivo. Así, el foco de interés de los investigadores de este campo no recae sobre las cogniciones individuales, sino en la manifestación colectiva del consenso.

El consenso estratégico, atributo de los equipos de trabajo, puede ser descrito a partir de aportes dados por diversos autores, como la comprensión compartida entre actores de la organización respecto de 
las prioridades estratégicas definidas en un contexto de trabajo, englobando las relaciones entre las prioridades competitivas y las políticas operacionales (Boyer \& McDermott, 1999; González-Benito, Aguinis, Boyd \& Suárez-González, 2010; Homburg, Krohmer \& Workman, 1999; Kellermanns et al., 2005).

En este punto, cabe mencionar que esa concepción es más reciente. En los primordios del uso de la expresión consenso estratégico, el foco de interés recaía en el grupo de gestores de la alta dirección, quienes tenían en sus manos la responsabilidad de decidir las macroestrategias de la organización (Kellermans et al., 2005). Posteriormente, la comprensión del término fue siendo ampliada y actualmente se entiende que, a pesar de ser los gestores de primera línea los responsables por las grandes decisiones, la implementación práctica de esas directrices estratégicas queda a cargo de mandos medios y de miembros de equipos, que son los responsables de la ejecución de las actividades. Esta perspectiva es defendida por Walter y colaboradores (2013), quienes manifiestan que el locus del consenso abarca tanto el nivel más elevado, como el medio y hasta el operacional de la organización. También se destaca el trabajo de Mahto y Davis (2012), quienes afirman que para el éxito organizacional es de gran importancia que los empleados del nivel operacional estén conscientes, no solo de las metas empresariales establecidas, sino también de los medios trazados para alcanzarlas. Más aún, esos autores defienden que la falta de concordancia entre los trabajadores de ese nivel puede poner en riesgo el alcance de las metas organizacionales.

A partir de esas consideraciones, el consenso estratégico es entendido como el acuerdo existente entre miembros del equipo en cuanto a los caminos definidos para alcanzar las metas de trabajo establecidas. Esos caminos son aquí entendidos como estrategias, lo que justifica el uso de la denominación consenso estratégico. Así, ese término se refiere a lo que el equipo ha de hacer, en términos de trabajo, para alcanzar los resultados esperados.

A pesar del acuerdo observado entre teóricos del área en cuanto al locus en que el consenso estratégico se aplica (miembros del equipo), aún subsisten discusiones cuando el asunto es el contenido del consenso. 
Alguno autores explicitan que es diferente el acuerdo en cuanto a la estrategia establecida para alcanzar la meta de trabajo, de aquella relacionada al proceso de toma de decisiones (Bowman \& Ambrosini, 1997; Dooley, Fryxelle \& Judge, 2000; Kellermanns et al., 2005; Mohammed \& Ringseis, 2001). En el primer caso se hace referencia al llamado consenso estratégico, fenómeno de interés de este estudio, y de cuya presencia se esperan resultados favorables para el quipo. En el segundo caso se habla de la búsqueda de acuerdo al momento de tomar la decisión, también llamada decisión forzada. Su presencia se asocia a decisiones más pobres, ya que lo que el grupo buscaría no seria la mejor solución, sino conseguir un acuerdo de los miembros, aun si fuera con relación a una decisión pobre (acuerdo forzado).

En el presente estudio, el foco de interés se encuentra en el acuerdo de los miembros del equipo en cuanto a los caminos establecidos para alcanzar las metas, y no a la forma como se toman las decisiones. Se entiende que la descripción hecha es superficial, pero la naturaleza de la segunda concepción escapa al foco de este trabajo, así, es presentado apenas para delimitar las bases teóricas que subyacen a este estudio empírico. También se debe mencionar que la importancia de la distinción referida está en el hecho de que la búsqueda de consenso, en el proceso de tomar decisiones, está prioritariamente asociada a resultados pobres del equipo, mientras que el consenso con relación a las estrategias ha sido mostrado como atributo capaz de predecir resultados positivos; sin embargo se puede evidenciar inconsistencia de ciertos hallazgos.

Con relación a la disimilitud de resultados antes referida, la revisión de estudios empíricos de este campo muestra algunas posibles explicaciones. Una de ellas es la propia definición del constructo, pues se observan concepciones dispares en los trabajos publicados sobre este tema. También se constata que existen distintos contenidos del consenso investigado; así, por ejemplo, en unos estudios se trabajó con el consenso sobre las tareas cotidianas, y en otros se midió el consenso sobre las macroestrategias. Adicionalmente, la revisión empírica revela que se adoptaron diferentes herramientas de medida y procesos de análisis, lo que torna muchos resultados no comparables, considerando que sus 
contenidos, a pesar del nombre similar, abordan fenómenos diferentes (Bowman \& Ambrosini, 1997; Dess \& Priem, 1995; González-Benito et al., 2010; Homburg et al., 1999; Kellermanns et al., 2005). Sobre la inconsistencia de resultados encontrados, Kellermanns y colaboradores (2011) destacan que parte de ella deriva de la presencia de moderadores de la relación entre consenso y desempeño, los cuales no siempre son considerados o tienen sus efectos controlados. Los autores sustentan su afirmación recurriendo a resultados de investigaciones que contenían o no moderadores. Analizando esos resultados, encontraron diferencias dependiendo de la presencia o no de las variables antes mencionadas. Discutiendo el papel del consenso en estudios empíricos, Mohamed y Ringseis (2001) sugieren que es pertinente suponer que en contextos estables sería más benéfico un consenso elevado, y en otros inestables sería necesario un consenso más bajo. Por lo tanto, existiría un nivel óptimo de consenso para cada situación, lo que significa incluir variables contextuales en los estudios de campo.

A pesar de esas limitaciones, se busca condensar algunos hallazgos que tornan evidente el papel del consenso en las organizaciones. Así, algunas investigaciones empíricas han conseguido demostrar que su presencia está acompañada de aumento de la cooperación, cohesión del grupo y de la habilidad de alcanzar nuevamente consenso, en futuras oportunidades (Kellermanns et al., 2005). También se ha verificado que la presencia del consenso lleva a los miembros del equipo a dejar de lado sus preferencias individuales y a adherirse a reglas de trabajo y caminos previamente establecidos por su grupo (Kellermanns et al., 2011). Por lo tanto, se trata de obedecer a reglas globales y colectivas, dejando menos espacio para que deseos personales y anhelos políticos tomen la delantera al momento de ejecutar las tareas.

La revisión de estudios empíricos también muestra que se ha buscado relacionar atributos individuales y grupales con el consenso estratégico. En ese sentido, cabe referir el trabajo de Knight y colaboradores (1999), quienes constataron que había relación negativa entre diversidad funcional y educación, por un lado, y el consenso estratégico, por otro. La diversidad de antigüedad en la empresa, por el contrario, 
presentó relación positiva con el consenso. Sin embargo, algunas de esas relaciones no fueron directas, pues variables como el conflicto interpersonal desempeñaron el papel de mediadoras parciales de esa relación, lo que resultó en un efecto intensificado. Trabajo similar fue realizado por Camelo, Fernandez-Alles y Hernandez (2010), quienes evidenciaron que el consenso estratégico desempeńa el papel de variable moderadora de la relación entre atributos individuales, como el nivel de educación y el desempeño innovador. Estos resultados, si bien no pueden ser vistos como evidencias definitivas, revelan la asociación entre atributos de nivel individual y el surgimiento del consenso, sea mediante relaciones directas o con la participación de elementos moderadores.

El trabajo de Mohammed y Ringseis (2001) estableció como foco de interés el estudio de los antecedentes y correlatos del consenso cognitivo. Las autoras verificaron que grupos que buscan unanimidad decisoria consiguen también mayor consenso cognitivo que los grupos que adoptan como método de decisión el voto de la mayoría. Sin embargo, grupos en los que hubo miembros más cooperativos no presentaron mayor disposición para alcanzar el consenso. Al investigar las consecuencias del consenso, las autoras constataron que los grupos con mayor nivel de consenso demostraron también mayor satisfacción con las decisiones que fueron tomadas. Tocando específicamente la eficiencia de las decisiones, Roberto (2004) observó que promover la participación de los funcionarios en las discusiones se mostró como una práctica asociada a un mayor consenso, así como la existencia de un líder menos directivo. Por otro lado, el trabajo realizado por Carney (2007) reveló que, en la medida en que los trabajadores se sienten involucrados con la organización, mayor consenso puede ser esperado.

Cambiando el análisis al nivel de la organización, se encuentra el estudio realizado por Walter y colaboradores (2013). Estos autores levantaron hipótesis relativas al impacto directo del consenso sobre los resultados organizacionales, así como esperaron que esa relación fuera moderada por una tercera variable denominada alineamiento estratégico, que envuelve el ajuste entre la organización y su medio ambiente. Los autores constataron que el consenso demostró tener un 
impacto directo sobre criterios declarativos de desempeño adoptados en esa investigación, y observaron la participación de la variable moderadora, la cual se hizo presente intensificando la relación cuando el alineamiento estratégico era elevado.

Teniendo como foco el desempeño organizacional, el estudio de Dess (1987) reveló que empresas con mejor desempeño presentaron consenso sobre los métodos, mas no sobre las metas. Las organizaciones con desempeños más pobres fueron aquellas en las que se observó un elevado acuerdo en relación con las metas, aunque hubo desacuerdo sobre los métodos.

El trabajo de González-Benito y colaboradores (2010) buscó investigar la relación entre el consenso sobre los medios y objetivos de trabajo, el desempeño y el dinamismo ambiental en empresas españolas manufactureras. Los resultados mostraron que la relación entre el consenso sobre los medios y el desempeño se ve alterada (moderada) por el dinamismo ambiental y es mediada por el consenso sobre los objetivos. Según esos autores, el estudio demuestra que la relación entre el consenso sobre los métodos y el desempeño es positiva en ambientes menos dinámicos y es negativa si ocurre en ambientes más dinámicos. Una investigación en un escenario similar fue realizada con una muestra de empresas mexicanas, también manufactureras, por RamosGarza (2009). Los resultados del trabajo de estos autores revelaron una relación positiva entre el consenso y el desempeño, observándose que esa relación fue moderada por la complejidad ambiental. Sin embargo, en este caso, los hallazgos poseen sentido invertido: bajos niveles de consenso estuvieron asociados a mejores desempeńos si el ambiente era de baja complejidad; contrariamente, en ambientes de elevada complejidad, el consenso mostró relaciones positivas con el desempeño alcanzado. De esta forma, los resultados de los estudios realizados en una muestra de empresas españolas y mexicanas revelan un papel diferenciado del ambiente, que en ambos casos fue definida como variable moderadora.

Como puede ser visto por los relatos de resultados de estudios empíricos realizados, el interés por la comprensión del papel del consenso 
estratégico continúa presente en las publicaciones del área, a pesar de los resultados son diversos y muchas veces contradictorios. Sobre ese hecho, Kellermanns y colaboradores (2011) afirman que es necesaria una mejor comprensión general de lo que es el consenso estratégico, así como de la relación de ese fenómeno con el desempeño. Para cumplir ese cometido, los autores afirman que la realización de estudios empíricos es el mejor camino, y complementan diciendo que existen evidencias del hecho de que el consenso estratégico impacta en diversos criterios de desempeño. Si se analizan los estudios publicados, se observará que ese tipo de relación se replica en investigaciones realizadas al nivel individual, grupal y organizacional. Por esa razón, se considera una contribución relevante el desarrollo de un trabajo que, centrándose en equipos de trabajo, indague el efecto del consenso sobre indicadores de desempeño.

Uno de los cuestionamientos más frecuentes en el campo de la psicología organizacional es el uso de la misma fuente para obtener informaciones tanto sobre la variable antecedente como sobre el criterio, lo que fragiliza los resultados, pues las asociaciones encontradas pueden resultar de la presencia de sesgo de fuente común. Al respecto, Spector (1994) reconoce la pertinencia de las críticas de investigadores en cuanto al uso de fuente única para recoger datos relativos a los trabajadores y al propio trabajo, cuando ambas son variables antecedentes y criterio del mismo estudio. Más aún, justifica la necesidad de mantenerse escéptico frente a resultados de trabajos que usaron, como estrategias para recoger datos, de instrumentos de autorreporte. Sin embargo, en una publicación posterior, el mismo autor (Spector, 2006) defiende que para capturar informaciones sobre estados subjetivos del participante, el autorrelato es una fuente legítima de información, pues la variable de interés. En esos casos, es la opinión del propio sujeto sobre un determinado objeto o evento, buscándose acceder a sus sentimientos, estados afectivos, emocionales o similares. En la publicación más reciente se menciona que para evaluar fenómenos visibles, externalizados o que se traducen en comportamientos observables, sería pertinente adoptar una segunda fuente de información. La misma recomendación se hace cuando una de las variables del estudio es un 
atributo de la organización, y no existe interés por saber la opinión de los trabajadores.

Teniendo en cuenta las observaciones de Spector (1994, 2006), el presente estudio que ahora relatamos, se buscó evitar el sesgo que resulta del empleo de fuente común (autorrelato), recogiendo los datos de interés de diferentes fuentes. Así, considerando la contribución teórica antes mencionada, se busca escapar de las fragilidades de método y hacer también una contribución en ese aspecto, tornando el estudio, en cierta medida, más confiable. Las especificidades de la realización empírica de la investigación propuesta son reportadas en la sección a seguir.

\section{Método}

\section{Participantes}

Participaron profesores y coordinadores de 70 planteles educativos. En cuanto a los docentes, ellos respondieron al instrumento de prácticas pedagógicas que cuestionaban sobre las estrategias de trabajo adoptadas para alcanzar las metas de trabajo establecidas, las cuales estaban relacionadas a la enseñanza. En total, 276 profesores participaron y todos ellos eran miembros de equipos pedagógicos de las instituciones educativas. Como tal, tenían metas de trabajo colectivas cuya consecución era vista como de responsabilidad compartida. Por otro lado, también se entregaron cuestionarios a 70 coordinadores pedagógicos de esas instituciones y se recibieron respuestas de 60 de ellos. En lo relacionado al tipo de escuelas, debe ser destacado que la muestra contempló tanto instituciones particulares como fiscales, siendo del tipo laico y religioso. No se descartaron cuestionarios por ausencia de respuestas o invariabilidad de ellas.

Las características funcionales y demográficas de la muestra de docentes y sus vinculaciones organizacionales pueden ser descritas como tratándose de un grupo compuesto, en su mayoría, por participantes de sexo masculino (61.6\%), con una media de edad de 42.1 
años $(D E=10.5$ años $)$ y educación mayoritariamente de nivel superior completo $(27.9 \%)$, especialmente en áreas pedagógicas $(35.5 \%)$, con una pequeña cantidad que posee algún tipo de curso de posgrado (17.6\%). En relación a la vinculación institucional, se observó que la mayor parte era de instituciones particulares laicas (59.8\%), la mitad de los participantes del estudio tienen 4 años o menos de antigüedad en el plantel (año de la mediana 2009) y trabajan en equipos relativamente pequeños (media aritmética $=16$ personas), probablemente influenciado por el hecho de que la mayor parte de la muestra sea originaria de instituciones particulares.

Con relación al grupo de coordinadores, la mayor parte fue del sexo femenino (55\%), y con curso superior en el área de Pedagogía (37.3\%), seguido del grupo que tenía algún curso de posgrado (28.3\%). La media aritmética de edad fue de 42.9 años, y con escasa diversidad de este atributo $(D E=9.6)$. Con relación a la antigüedad en el plantel educativo, el año que aparece como mediana fue 2007. Esos datos permiten describir este grupo genéricamente como compuesto de mujeres, de media edad, con elevado nivel de educación, y que se encuentran hace por lo menos 7 años en la institución, en la época en que los datos fueron recogidos (año 2013).

\section{Medidas e instrumentos de medición}

Teniendo en cuenta que la fiabilidad de los instrumentos utilizados es condición imprescindible para el inicio de la etapa empírica de una investigación, se tomó la decisión de emplear herramientas de medida con evidencias de validez previamente comprobadas en estudios anteriores, en grupos con culturas similares a la de este estudio, sabiendo que las evidencias de fiabilidad pueden ser alteradas conforme cambian las características de la muestra en que se recogen los datos.

Consenso estratégico: escala compuesta por 14 afirmaciones relativas a las estrategias de trabajo definidas en la institución educativa en que trabajan. Las preguntas fueron respondidas en escala del tipo Likert de concordancia de 5 puntos y con índices da fiabilidad satisfactorios (Alfa de Cronbach $=.92$; correlación ítem total $=.66$ ) una muestra de 
docentes ecuatorianos (Puente-Palacios \& Puente, 2013). Fue respondida por los miembros del equipo pedagógico y por el coordinador del plantel. Estos últimos respondieron refiriéndose a su concordancia sobre las estrategias de trabajo del equipo docente del plantel que coordinan. Cuando es respondida por el coordinador, la variable es denominada de Prácticas pedagógicas (evaluación de).

Compromiso afectivo: medido a partir de 9 afirmaciones respondidas en escala tipo Likert de concordancia de 7 puntos (Alfa de Cronbach = .91; correlación ítem total $=.75$ ). Hubo pérdida de 2 ítems por contribución pobre (carga factorial), razón por la cuál los análisis se hicieron con los datos ofrecidos por 7 de las 9 preguntas. Esta medida fue respondida por los miembros del equipo pedagógico y por el coordinador.

Satisfacción con el equipo: considerada criterio de efectividad, según Hackman (1987), fue evaluada por 5 afirmativas respondidas en escala de concordancia ajustada a sentimientos (la afirmación no refleja mis sentimientos, o, la afirmación refleja mis sentimientos). Las evidencias de fiabilidad para la muestra ecuatoriana fueron satisfactorias (Alfa de Cronbach $=.90$; correlación ítem total $=.77$ ). También este instrumento fue respondido por los miembros del equipo pedagógico, así como por el coordinador.

Desempeño del equipo: evaluado utilizando una medida de criterio de evaluación razonada o criterio de juicio sobre el desempeño. Fue calificado mediante el uso de una escala compuesta de 9 frases que describen el desempeño del equipo. Cada frase fue respondida en una escala del tipo Likert de 7 puntos (Alfa de Cronbach $=.93$; correlación ítem total $=.76$ ). Las respuestas fueron obtenidas tanto del coordinador (hetero-evaluación) del plantel, como por los miembros del equipo pedagógico (autoevaluación).

\section{Procedimiento}

Para tener acceso a los participantes del estudio, los miembros del equipo de investigación visitaron los establecimientos educacionales, cuyos nombres serán mantenidos en sigilo, respetando el derecho al anonimato, y entraron en contacto el coordinador o director, quien 
autorizó la recogida de los datos. Una vez autorizados, se definió el día en que los cuestionarios serían aplicados. Al momento de distribuir los cuestionarios entre los docentes, fue explicado el objetivo y la naturaleza de la participación, con el propósito de demostrar la forma cómo deberían proceder para responderlos. También fue destacado que la participación era voluntaria, y que los participantes serían protegidos por el anonimato, razón por la cual aquellos que decidían participar no deberían colocar su nombre o identificarse de cualquier forma. Posteriormente, fue enfatizado que los datos recogidos serían analizados de forma agrupada y sin permitir al plantel educativo el acceso a las respuestas individuales. Con la adopción de estos procedimientos fueron preservados los preceptos de la ética en investigaciones científicas. La información relativa al estudio, así como el carácter voluntario, constaban como carátula del conjunto de cuestionarios que se entregaron.

Los datos recogidos fueron tabulados en el paquete de análisis estadísticos SPSS, versión 18.0. Estos fueron analizados considerando el objetivo establecido para la realización del estudio, pautado en un modelo explicativo que indaga el poder de predicción del consenso estratégico en relación con el desempeño de equipos. Por tanto, luego de constatar la fiabilidad de los instrumentos utilizados, investigada según las orientaciones ofrecidas por Tabachnik y Fidel (2007), se procedió a la verificación de la pertinencia de tratar los datos recogidos de los miembros de los planteles como informaciones genuinamente relativas a sus equipos. Se verificó si ocurría el proceso emersión del nivel individual al colectivo, del consenso estratégico, el desempeño, el compromiso y la satisfacción.

La posibilidad de emersión fue investigada mediante el cálculo de la magnitud de la desviación estándar, técnica referida por Kellermanns y colaboradores (2011) como mecanismos de verificación del nivel de semejanza de las respuestas de miembros del grupo. De la misma forma lo hacen Tarakci y otros (2014) quienes afirman que la desviación estándar es usualmente adoptada como indicador del consenso en equipos. La desviación estándar revela la magnitud de la heterogeneidad de las respuestas individuales, que si es escasa (menor que 25\% del valor 
de la media aritmética) muestra semejanzas en las respuestas. La lógica subyacente es que si las respuestas de los miembros del equipo son similares, entonces el investigador está frente a un atributo colectivo.

Por otro lado, para la constatación de la emersión también es necesario verificar la existencia de diferencias significativas entre los grupos, en este caso, planteles educativos. Para ello se calculó una ANOVA y se verificó la magnitud de la correlación intraclase (ICC-Itracalss correlation) para todas las variables del estudio. En el caso de la ANOVA se necesita un resultado estadísticamente significativo y en el caso del ICC, es necesario constatar la existencia de varianza entre grupos que atestigüe la presencia de diferentes patrones de respuestas. En la medida en que los dos criterios (similitud dentro del grupo y diferencias entre grupos) se muestran satisfactorios, es pertinente concluir sobre la manifestación del proceso de emersión, y proceder a la construcción de puntajes grupales, o en este caso, puntuaciones por cada plantel educativo.

Una vez creados esas puntuaciones, luego de verificar que los criterios previstos fueron satisfechos para todas las variables del estudio, se procedió a la construcción del modelo de predicción, utilizando para tanto una regresión linear que permite obtener respuestas para las preguntas de investigación levantadas. Los resultados obtenidos al verificar el papel predictivo del consenso son descritos a seguir.

\section{Resultados}

Conforme anunciado, en primer lugar se investigaron las evidencias de fiabilidad estadística de las escalas usadas. En el caso del consenso estratégico cabe recordar que este deriva de la concordancia de los miembros del equipo cuanto a las prácticas pedagógicas (estrategias de trabajo). Esa medida, cuando fue respondida por los profesores de los planteles educativos, mostró una estructura unifactorial, con cargas adecuadas (entre .49 y .72) y mediante la que se consiguió capturar aproximadamente $43 \%$ de explicación de la varianza $(\alpha=.90$; 
$r$ ítem-total $=.58)$. En el caso de los coordinadores, la misma escala revela su opinión sobre las llamadas prácticas pedagógicas, mas no pudo tener su solución factorial investigada, en razón del reducido número de participantes (60) comparado a la cantidad de ítems. Sobre el tamaño necesario de la muestra para la realización de un análisis factorial, Pasquali (1999) señala un valor mínimo de 5 respuestas por ítem, relación que no se consiguió porque esta escala posee 14 preguntas. Por esa razón, se verificaron directamente sus índices de fiabilidad, que se mostraron satisfactorios $(\alpha=.92 ; r$ ítem-total $=.64)$.

Procedimientos similares fueron adoptados para las demás escalas, que se revelaron adecuadas. Con relación a los cuestionarios respondidos por los profesores, el de satisfacción presentó solución unifactorial con cargas elevadas (entre .70 y .90), explicó $73.3 \%$ de varianza y mostró ser una medida apropiada para evaluar el fenómeno de interés al revelar una consistencia interna satisfactoria $(\alpha=.91 ; r$ ítem-total $=.77)$. En cuanto a la medida de compromiso o vínculo afectivo con el equipo, también de estructura unifactorial, se constató que dos ítems de contenido negativo presentaron una contribución pobre (carga factorial $<$.30) y por esa razón fueron excluidos. Tomada esa decisión, la medida quedó compuesta por 7 ítems que se congregan en un factor único que captura $67.3 \%$ de la varianza, muestra cargas factoriales elevadas (entre .61 y .88), y tiene índices adecuados de confiabilidad interna $(\alpha=.92$; $r$ ítem-total $=.75$ ).

Las evidencias estadísticas de fiabilidad, así como la estructura factorial, pudieron ser calculadas para las medidas de compromiso y satisfacción, cuando fueron respondidas por los coordinadores, pues poseen un número bajo de ítems. Así, en el caso de los coordinadores, la escala de satisfacción mostró estructura también unifactorial, con cargas entre .34 y .84 , consiguió capturar $67 \%$ de la varianza, obtuvo un valor de Alfa de Cronbach de .88 y el valor medio de la correlación ítem-total fue de .71. Ya en caso de la escala de compromiso afectivo con el equipo, los valores de las cargas oscilaron entre .89 y .66 y también fueron retirados los dos ítems negativos, por sus contribuciones pobres para la medida. El conjunto de 7 ítems se agregó en un factor 
único que consiguió capturar $67.74 \%$ de varianza y mostró índices de fiabilidad adecuados $(\alpha=.91 ; r$ ítem-total $=.74)$.

La escala de evaluación del desempeño del equipo fue respondida por los profesores y por el coordinador pedagógico, de tal forma que se contó tanto con autorreporte como con hetero-reporte para la variable criterio del estudio. La decisión de adoptar ese procedimiento surgió de la necesidad de minimizar la contaminación resultante de fuente única como base de información de las variables antecedentes y consecuentes. En ambas muestras (profesores y coordinadores), la solución encontrada para la escala fue unifactorial, y para el caso de los docentes, las nueve afirmaciones se estructuraron un factor que capturó $65.84 \%$ de la varianza y mostró índices de consistencia interna satisfactorios $(\alpha=.93 r$ ítem-total $=.76)$. Por otro lado, en el caso de la evaluación de desempeño hecha por los coordinadores, aunque el tamaño de la muestra es reducido, aún está dentro del tamaño mínimo que según Pasquali (1999) permite ejecutar un análisis factorial. Así, se observó que la estructura unifactorial consigue explicar $70.76 \%$ de la varianza y posee índices también adecuados de confiabilidad $(\alpha=.91 ; r$ ítemtotal $=.78)$.

Una vez constatada la adecuación de los instrumentos a la muestra de participantes con la que el estudio fue realizado, se dio continuidad a la verificación de la emersión de los fenómenos contemplados en la investigación. Esto es, se analizó en qué medida era posible construir escores grupales a partir de respuestas individuales. Ese tipo de análisis es una exigencia en este tipo de investigación, puesto que las variables son del nivel del equipo, mas los datos fueron recogidos del nivel individual, es decir, de las personas que componen esos equipos. También es importante destacar que no es suficiente condensar los puntajes individuales para componer variables grupales. De acuerdo con Chan (1998), los fenómenos colectivos capturados a partir de respuestas individuales deben demostrar padrones de semejanza intragrupal que justifiquen el hecho de ser considerados atributos colectivos. Por tanto, es necesario adoptar estrategias analíticas que muestran que los datos se comportan de tal forma que la agregación está autorizada. 
Buscando atender a esa exigencia, dos tipos de análisis fueron necesarios. En primer lugar, se verificó la semejanza de las respuestas entre profesores y, a continuación, se investigaron las diferencias de respuestas entre planteles. Para ejecutar el análisis de las respuestas de los profesores se procedió al cálculo de la magnitud de la desviación estándar (o desviación típica) y cabe recordar que valores bajos revelan elevada semejanza. La adecuación del uso de este método es defendida por Kellermanns y colaboradores (2011), quienes relatan que una de las técnicas utilizadas en las investigaciones sobre consenso estratégico para verificar la semejanza de respuestas de los miembros del grupo es el cálculo de la magnitud de la desviación típica. Los valores encontrados en este estudio fueron de .48 para prácticas pedagógicas (media aritmética $=4.31) ; .98$ para compromiso afectivo, (media aritmética $=5.73)$; .76 para satisfacción (media aritmética $=4.09$ ); y .89 para desempeño del equipo (media aritmética $=5.84$ ). En el caso de la escala de desempeño respondida por el coordinador, este tipo de análisis no fue necesario, pues había una única respuesta por plantel educativo, por tanto, no había desviación estándar a ser calculada.

El análisis del significado de esos valores debe hacerse por comparación con el valor de la media aritmética, lo que resulta en el llamado índice de variación. Cuanto menor es ese índice, más similares son las respuestas individuales. Como puede ser observado, el valor máximo es de la variable satisfacción que corresponde a $18.58 \%$ de la media aritmética. Si se considera que valores alrededor de $24 \%$ son asumidos como evidencias de semejanza de respuestas, aquellos obtenidos en este estudio permiten afirmar que, en relación a las variables recogidas, se observa un mismo padrón de respuestas individuales. De estos valores, uno de los más importantes es el del consenso estratégico, variable central del estudio, por esa razón se ofrecen informaciones adicionales.

En cuanto a su comportamiento, se verificó no solo la desviación típica general (en toda la muestra), sino que examinó también por cada plantel educativo, pues en todos se esperaba encontrar elevado consenso. Los resultado observados fueron desviaciones, por escuelas, entre $.07 \mathrm{y}$ .97. Con esos datos y los respectivos valores de las medias aritméticas 
se calculó el índice de variación y se obtuvo valores entre 1.47 y $22.6 \%$ de variación, lo que demuestra elevado consenso o baja discrepancia de respuestas.

Luego, se procedió con el segundo tipo de análisis que es relativo a la verificación de existencia de diferencias entre planteles. Para ello, fue calculada una ANOVA (one way) buscando comparar las escuelas en relación con las variables antes citadas. Los resultados encontrados mostraron diferencias significativas $(p<.05)$ para tres de las cuatro variables. El compromiso mostró diferencia marginal, pues el valor $p$ fue superior a lo esperado $(p=.08)$. Entretanto, considerando que la magnitud de la probabilidad de error no supera el 10\%, se tomó la decisión de mantenerlo en el estudio, recordando que las diferencias entre planteles, en relación con esta variable son marginales (según principios estadísticos). Un segundo criterio de evaluación de la existencia de diferencias significativas entre planteles, en relación a las variables bajo análisis, fue utilizado buscando corroborar los datos revelados por la ANOVA. Así, se calculó el coeficiente de correlación intraclase (ICC). Los valores encontrados fueron de ICC = .17 para satisfacción, ICC $=.11$ para desempeño evaluado por auto-relato, ICC $=.18$ para consenso estratégico e ICC $=.07$ para compromiso. Para interpretar esos valores se recurrió a las orientaciones de Bliese (2000) quien, sustentado en teóricos del área, afirma que valores típicos en el área organizacional están entre 0 y .5 (mediana de .12) y, al relatar sus propios hallazgos, afirma no haber encontrado valores de ICC mayores que .3.

Con esos parámetros se puede afirmar que, en el caso de las variables recogidas de los miembros de los equipos pedagógicos, se observaron diferencias cuya magnitud es compatible con los valores típicos del área, con la excepción de la variable compromiso, cuyo valor nuevamente es ligeramente inferior al de la mediana relatado por otros autores, más aún así revela la presencia de diferencias entre equipos. Con relación al compromiso, se debe recordar también que no es foco de central interés del modelo que se está analizando, pero su presencia en el estudio se justifica por la necesidad de controlar el efecto de la varianza que comparte con la variable criterio que es el desempeño del equipo. 
Los resultados antes relatados muestran que el conjunto de datos recogidos de los miembros de los planteles educativos, en relación a la satisfacción, consenso estratégico, desempeño y compromiso, se comportan como atributos de los equipos, pues las respuestas dadas por los profesores de las mismas escuelas fueron semejantes al interior de cada escuela (evidencia dada por la desviación típica) y, al mismo tiempo, fueron diferentes de las respuestas medias dadas por los de otras escuelas (evidencias de la Anova e ICC). Sobre la base de esto fueron construidos puntajes que representan el comportamiento de las variables por escuelas. Así, el banco de datos con que se trabaja de aquí en adelante está compuesto por 60 planteles educacionales y con él se construyó el modelo de predicción que busca identificar el poder de explicación del consenso estratégico, en relación al desempeño del equipo.

Para dar inicio a la investigación del modelo subyacente al estudio propuesto, se verificaron los presupuestos de la regresión linear, técnica a ser usada en esta parte de la investigación. Buscando identificar el patrón de distribución de las respuestas en las diferentes variables, se calculó la magnitud de la asimetría de la distribución de las respuestas empíricas en relación a la distribución normal. Los resultados se mostraron adecuados, según Miles y Shevlin (2001), con valores de asimetría superiores a 1 que muestran un ligero distanciamiento de la curva normal, más no exigen intervención. Sin embargo, los mismos autores mencionan que valores superiores a 2 (en términos absolutos) demandan transformación de los datos para forzar la normalidad necesaria y así poder dar continuidad a los análisis paramétricos. Al analizar la distribución de las respuestas, por variable, se encontraron valores de asimetría mayoritariamente distribuidos entre -.28 y - -.90 . En el caso del desempeño del equipo evaluado por el coordinador, se observó un valor de 1.50 . Sobre este dato, ha de ser mencionado que es inferior al definido por los autores antes referidos como problemático (2.0 en valores absolutos). Por esa razón, se tomó la decisión de no transformar esa variable y proseguir con los análisis necesarios para la evaluación de la pertinencia del modelo de predicción propuesto. 
Dando continuidad al análisis de los presupuestos, se realizó la inspección de la matriz de correlaciones entre las variables. La Tabla 1 muestra los resultados encontrados y evidencia asociaciones significativas entre la mayoría de ellas. Al mismo tiempo, se observa la ausencia de indicios que sugieran redundancia de variables. Se destaca el valor de la relación entre la evaluación que el coordinador hace sobre las prácticas pedagógicas del equipo docente y su evaluación sobre el desempeño del equipo (.80). Ese resultado revela la adecuación de haber tomado la decisión de recoger los datos de las variables antecedentes y consecuentes de fuentes diferentes. También cabe destacar que la misma relación no se observa al analizar la asociación existente entre el desempeño evaluado por los miembros del equipo y su concordancia sobre las estrategias de trabajo (consenso estratégico). Ese dato permite inferir que ambos fenómenos son relacionados, aunque no son iguales.

\section{Tabla 1}

\section{Correlaciones entre las variables del estudio}

\begin{tabular}{lccccccc}
\hline Variables & 2 & 3 & 4 & 5 & 6 & 7 & $8^{*}$ \\
\hline Consenso Estratégico P. & .05 & .51 & .39 & .21 & .03 & .05 & .28 \\
Compromiso P. & & .75 & .77 & .19 & .09 & .02 & .22 \\
Satisfacción P & & & .74 & .16 & .04 & -.00 & .28 \\
Desempeño P. & & & & .28 & .16 & .16 & .40 \\
Prácticas Pedagógicas C. & & & & & .69 & .64 & .80 \\
Compromiso C. & & & & & & .75 & .67 \\
Satisfacción C & & & & & & & .78 \\
\hline
\end{tabular}

Nota: ${ }^{*}$ Desempeño del equipo evaluado por el Coordinador; P.- Profesores; C.- Coordinador; $r$ significativas $\geq .28$ ( $p \leq .05)$.

Los datos de la tabla muestran adicionalmente relaciones significativas y de magnitud considerable entre la evaluación del desempeño, la satisfacción y el compromiso. Por tanto, sugieren la necesidad de controlar su efecto al construir los modelos de predicción. Luego de haber 
verificado el comportamiento de las variables del estudio, entendidas como representantes de atributos del nivel meso, se procedió a la construcción de las ecuaciones que engloban los términos relacionados en el modelo de investigación establecido. Para ello, se construyeron dos modelos en los cuales las variables antecedentes y criterio fueran recogidas de diferentes fuentes (profesores y coordinador). De esa forma, para la predicción del desempeño del equipo, y de la forma cómo es percibido por el coordinador, el consenso estratégico utilizado como variable explicativa fue el recogido de los miembros del equipo. Para la predicción del desempeño, conforme relatado por los miembros del equipo, la variable explicativa utilizada fue la ofrecida por el coordinador.

Sobre los datos con que se trabajó hay que recordar que el consenso estratégico es el predictor estudiado; entre tanto, cuando la información es recabada del coordinador, se adopta la denominación Prácticas Pedagógicas (evaluación de), pues en este caso no es posible verificar la existencia de consenso, considerando que existe un único coordinador por plantel. Así, esa denominación es el sinónimo de consenso estratégico y es usada cuando la información es dada por los coordinadores. También cabe enfatizar que el desempeño del equipo pedagógico, variable criterio del presente estudio, fue obtenido a partir de descripciones hechas por los miembros del grupo (autoevaluaciones), así como mediante el relato hecho por el coordinador (hetero-evaluación). Al momento de construir los modelos de predicción y considerando las correlaciones observadas entre las variables satisfacción, compromiso con el equipo y desempeño, ellas tuvieron su efecto controlado antes de introducir la variable consenso o evaluación de las prácticas, según fuera el caso.

En un primer momento se investigó el poder predictivo del consenso estratégico, como atributo del equipo, en relación a la evaluación del desempeño hecha por el coordinador. El modelo de predicción fue construido en dos pasos, con el fin de evidenciar el efecto del predictor después de retirado el efecto de la satisfacción y el compromiso con el equipo. Los resultados encontrados demostraron que la satisfacción y el compromiso del coordinador, con relación al equipo, impactan sobre la forma cómo él evalúa el desempeño. Asimismo, se observó que una 
vez controlado su efecto, el hecho de que los miembros del equipo concuerdan en relación con los caminos escogidos para alcanzar las metas de trabajo, o el llamado consenso estratégico, tiene un efecto adicional en la predicción del desempeño. Los principales resultados pueden ser vistos en la Tabla 2.

\section{Tabla 2}

Predicción del desempeño evaluado por el Coordinador, a partir del consenso estratégico

Variables antecedentes

Paso 1

Satisfacción C.

Compromiso C.

$\Delta^{2}$

$.62^{*}$

Paso 2

Consenso estratégico P.

$R^{2}$

$.68^{*}$

Nota: ${ }^{*} p<.01$.

Los datos mostrados demuestran que el hecho de que los miembros del equipo concordaran con las estrategias de trabajo definidas para alcanzar las metas, responde al 6\% de la evaluación del desempeño que el coordinador hace sobre el trabajo del equipo. Si se analiza los resultados integrales, se verifica que el $68 \%$ de ese desempeño es explicado por el modelo de investigación propuesto que condensa tanto la satisfacción como el compromiso evaluados por el coordinador y el consenso relatado por los miembros del equipo pedagógico. Por tanto, estos resultados corroboran la relación esperada entre el consenso estratégico y el desempeño. 
Dando continuidad con el estudio, se construyó una segunda ecuación, en la que se investigó el poder de predicción de las prácticas pedagógicas vistas por el coordinador, en relación con el desempeño del equipo, evaluado desde la perspectiva de los propios miembros, es decir, mediante autoevaluación. Los resultados obtenidos están dispuestos a continuación (ver Tabla 3).

\section{Tabla 3}

Predicción del desempeño del equipo, a partir de la evaluación del coordinador sobre las prácticas pedagógicas

Variables antecedentes

Paso 1

Satisfacción P.

Compromiso P.

$\Delta^{2}$

$.65^{*}$

Paso 2

Prácticas pedagógicas $\mathrm{C}$.

$R^{2}$ .66

Nota: ${ }^{*} p<.01$

Conforme el lector observa, la percepción del coordinador sobre las prácticas pedagógicas no tuvo poder de predicción sobre la evaluación que el equipo hizo sobre su propio desempeño. Adicionalmente, se aprecia que el poder predictivo de la satisfacción y del compromiso, que a pesar de ser significativos, no constituyen foco de interés de esta investigación y entraron en los modelos solamente para tener su efecto controlado. El conjunto de resultados obtenidos en el estudio revela que el consenso estratégico, como característica del equipo de trabajo, tiene el poder de predecir los resultados del trabajo del equipo. La discusión, mas detallada, de estos hallazgos y constataciones, se presenta en la siguiente sección. 


\section{Discusión}

El objetivo del estudio realizado fue investigar el poder explicativo del consenso estratégico sobre los resultados del desempeño. El sustento que da soporte a esa relación se basa en elaboraciones teóricas según las cuales el hecho de que los miembros de un equipo concuerden con las estrategias de trabajo definidas para que las metas sean alcanzadas es condición primera para que los miembros estén dispuestos a movilizar esfuerzos con miras a la realización de la tarea incumbida (Kellermanns et al., 2011; Tarakci et al., 2014).

Sin embargo, los resultados empíricos de estudios del área son inconsistentes, pues se han constatado relaciones directas, moderadas, curvilíneas o ausentes, lo que sirve como justificación para que la investigación sobre esta temática sea extendida, pues más evidencias sobre la naturaleza da la relación entre consenso estratégico y el desempeño aún son necesarias.

Una de las primeras consideraciones que deben hacerse sobre los hallazgos propiciados por esta investigación es relativa al uso de datos recogidos de los miembros del equipo para, a partir de ellos, derivar la variable de nivel meso o atributo del equipo, conocida en la literatura del área bajo la denominación de consenso estratégico. Los resultados obtenidos mostraron que hubo elevada semejanza de respuestas de los miembros de los planteles participantes del estudio, en cuanto al uso de estrategias que favorezcan el alcance de la meta de trabajo. La realización de un análisis pormenorizado, según plantel educativo, reveló la existencia de respuestas similares, por parte de los miembros, pues la discordancia máxima mostró un valor relativamente bajo $(22 \%$ en relación al tamaño del valor de la media aritmética). Ese valor muestra que los docentes de los planteles educacionales tendieron a mostrar opiniones semejantes y favorables sobre las estrategias usadas como guía para el trabajo que colectivamente ejecutan.

Paralelamente, la comparación entre establecimientos educativos mostró la presencia de diferencias relevantes (estadísticamente significantes) entre ellos, en relación al consenso estratégico, las cuales fueron 
evidenciadas a partir del cálculo de una ANOVA y de la verificación del tamańo del ICC. Con estos resultados es pertinente defender que el consenso estratégico, atributo de los equipos, puede ser recabado mediante el uso de una escala aplicada a los miembros del equipo. Posterior a la recogida de datos, el investigador deberá averiguar la magnitud de la semejanza de respuestas por equipo, así como las diferencias entre equipos, demostrando la existencia de homogeneidad interna y heterogeneidad externa, conforme a lo exigido para el estudio de fenómenos que se constituyen a partir de emersión, como defendido por Chan (1998).

Los patrones de respuestas identificados por escuelas (o equipos), muestran la ocurrencia del fenómeno de emersión, descrito como el surgimiento de un atributo colectivo que nace a partir de propiedades individuales. La importancia de esa constatación está en el hecho de que diversos procesos grupales no pueden ser capturados directamente del nivel colectivo, lo que exige que se levanten datos de los individuos. En esas circunstancias, sin embargo, queda la duda sobre el hecho de haber capturado un atributo grupal. En cuanto a esa duda, el estudio realizado mostró las técnicas que pueden ser adoptadas para disiparla y probar empíricamente que, a partir de las respuestas de los miembros, se pueden derivar atributos colectivos, que dan evidencias de la semejanza intragrupal y diferencias intergrupales.

Respecto de las relaciones significativas encontradas entre consenso estratégico y desempeńo, los resultados de este estudio guardan semejanza a otros similares llevados a cabo en diversos campos. Si se analiza la magnitud del efecto reportado por Kellermanns y colaboradores (2011) en su estudio meta-analítico, y se compara con el poder explicativo del consenso en este estudio, se observa que es de menor magnitud, considerando que el consenso explica 6\% de la varianza del desempeño del equipo, frente a los reportados por esos autores que se ubicaron entre 18 y $22 \%$. Sin embargo, ese relato también mostró que al analizar la credibilidad de los resultados reportados, a un intervalo de $80 \%$ de confianza, valores de correlaciones iguales a cero estaban presentes. Por tanto, a pesar de observar un poder de predicción mayor, también se encontra- 
ron estudios donde hubo ausencia de asociación entre esas variables. Así, la contribución de $6 \%$ del consenso estratégico para la predicción del desempeño no debe ser minimizada.

En el caso de la segunda ecuación de regresión construida, se observa la ausencia de poder explicativo de la percepción del coordinador sobre las prácticas pedagógicas. Esa ausencia de asociación muestra que el poder explicativo no resulta de la aprobación de las estratégicas de trabajo definidas por el equipo (como ocurrió cuando ellas fueron evaluadas por el coordinador). Más bien, resulta del hecho de que los miembros del equipo concuerden con esas prácticas, ya que la significación estadística solo aparece cuando el atributo que se investiga es realmente una propiedad del equipo (concordancia de los miembros), como ocurrió en la primera ecuación. Así, el poder de predicción es del consenso estratégico, y no de la evaluación de adecuación de las prácticas de trabajo adoptadas por el equipo.

Los resultados de los dos modelos de predicción traen evidencias adicionales a la literatura del área que establece la importancia del consenso estratégico para el buen funcionamiento del equipo de trabajo. Walter y colaboradores (2013) destacan que este tema ha sido estudiado por más de cuatro décadas. A pesar de la cantidad de investigaciones realizadas, al asunto no se ha agotado y los resultados reafirman su importancia en el contexto de los equipos de trabajo. La lógica subyacente al papel del consenso se sustenta en el hecho de que el mayor acuerdo entre los miembros del equipo favorece el desarrollo de acciones armoniosas, mejora de la comunicación, coordinación y en general, mejora el desempeño. A pesar del entusiasmo por los resultados identificados, la magnitud reducida sugiere que esa relación puede no ser directa. Así, se defiende la necesidad de inclusión de otras variables de proceso que pueden actuar como mediadoras o moderadoras de la relación entre consenso y desempeño. Hipotetizando relaciones indirectas Kellermanns y colaboradores (2011), y Walter y otros (2013) relatan la existencia de estudios que no consiguen evidenciar cualquier efecto a pesar de que la lógica teórica sustente su presencia. Eso, según los autores mencionados, puede deberse al hecho de que las investigaciones 
realizadas no incluyeron variables que actúen como correlatos u otras que con papeles diferenciados que pueden afectar la relación.

Sin embargo, y a pesar de lo dicho, los hallazgos del estudio relatado en este manuscrito no pueden tener su contribución analizada solo desde la perspectiva teórica, pues hay implicaciones prácticas que merecen ser consideradas. El conjunto de datos de los estudios de este campo sugiere que los gestores estén atentos al hecho de que los miembros de los equipos que comandan pueden no concordar con las estrategias de trabajo establecidas para alcanzar las metas. Si esas personas no están convencidas de que el plan de acción definido es un camino adecuado, la probabilidad de que ese equipo no tenga éxito es elevada. Adicionalmente, los resultados del estudio empírico revelan que el hecho de que el coordinador o líder del equipo, considere que las estrategias de trabajo del equipo son adecuadas, no es suficiente para obtener buenos resultados en el desempeño del equipo. Así, es pertinente defender que es necesaria la implicación de los miembros, con las estrategias definidas para el trabajo colectivo, para que sean alcanzados los resultados esperados.

En relación a la opinión de los miembros del equipo, los resultados alcanzados también revelan que un mayor consenso se asocia a mejores resultados o, en este caso, a mejor desempeño. Eso demuestra que no es suficiente que uno u otro miembro del equipo considere las estrategias de trabajo adecuadas. Es necesario que el conjunto de personas tenga esa misma visión, es decir, es necesario que haya consenso. De esa constatación podemos derivar la afirmación relativa a la necesidad de los gestores de equipos de cuidar del equipo como un todo, y no apenas de unos cuantos miembros si lo que se busca es la optimización del desempeño grupal.

Como todo estudio este también posee algunas limitaciones que necesitan ser reconocidas. Una de ellas es el reducido tamaño de la muestra, que si bien permite la utilización de técnicas de análisis paramétrico, también está asociada a la presencia de error estadístico de tamaño mayor si se compara con el que se puede observar en muestras que cuentan con más participantes. Una segunda limitación es el hecho 
de que el estudio fue realizado en un único tipo de organización, y al ser todos los datos recogidos de planteles de educación, un mismo patrón de respuesta puede estar presente. Por último, una tercera limitación refiere al uso de un método único para recabar las informaciones, que puede resultar en el sesgo de mono-método, que podría influenciar en la presencia de relaciones significativas. Sobre ese riesgo, entre tanto, la inspección de la matriz de correlaciones revela asociaciones de magnitud moderada y diferenciada, lo que permite defender que el método común no llegó a contaminar los datos de manera a invalidar los hallazgos reportados.

A pesar de esas limitaciones, cabe mencionar algunas contribuciones concretas del estudio realizado. Así, se inicia mencionando el hecho de ser muy poco común la presencia de estudios empíricos de nivel meso. Para la realización de esta investigación fueron recogidos datos de los individuos, y se utilizaron técnicas analíticas visando constatar la pertinencia de componer, a partir de ellos, puntajes grupales. Los análisis de predicción fueron realizados todos en el nivel de los equipos y eso constituye en aspecto diferencial del estudio. Una segunda contribución es el hecho de haber mostrado que el poder predictivo resulta del consenso estratégico del equipo sobre las prácticas pedagógicas y no de la evaluación que el coordinador hace sobre esas prácticas. Por último, el hecho de haber recurrido a diferentes fuentes para obtener informaciones de las variables antecedente y consecuente es un aspecto favorable, pues permite mayor confianza en los resultados, cuanto a la veracidad de las asociaciones identificadas.

A pesar de las contribuciones del estudio y de las cuatro décadas dedicadas por estudiosos del área a la compresión del papel del consenso estratégico en equipos de trabajo, hay que reconocer que el camino no ha terminado y existe una agenda de investigación aún por cumplir. Son necesarios más estudios, de preferencia de diseño longitudinal, que investiguen relaciones curvilíneas, y que consideren la participación de variables moderadoras y mediadoras de la relación entre el consenso estratégico y el desempeńo, para que sea posible llegar a conclusiones cabales sobre esta temática. 


\section{Referencias}

Bliese, P. D. (2000). Within-group agreement, non-independence and reliability. En K. J. Klein \& S. W. J. Kozlowski (Eds.), Multilevel theory, research and methods in organizations: Foundations, extensions and new directions (pp. 349-381). San Francisco, CA: Jossey-Bass.

Bowman, C. \& Ambrosini, V. (1997). Perceptions of strategic priorities, consensus, and firm performance. Journal of Management Studies, 34(2), 241-258.

Boyer, K. K. \& McDermott, C. (1999). Strategic consensus in operations strategy. Journal of Operations Management, 17(3), 289-305.

Camelo, C., Fernández-Alles M. \& Hernández, A. B. (2010). Strategic consensus, top management teams, and innovation performance. International Journal of Manpower, 31(6), 678-695.

Carney, M. (2007). How commitment and involvement influence the development of strategic consensus in health care organizations: The multidisciplinary approach. Journal of Nursing Management, 15(6), 649-658.

Chan, D. (1998). Functional relations among constructs in the same content domain at different levels of analysis: A typology of composition models. Journal of Applied Psychology, 83(2), 234-246.

Cohen, S. G. \& Bailey, D. E. (1997). What makes teams work: Group effectiveness research from the shop floor to the executive suite. Journal of Management, 23(3), 239-290.

Dess, G. G. (1987). Consensus on strategy formulation and organizational performance: Competitors in a fragmented industry. Strategic Management Journal, 8(3), 259-277.

Dess, G. G. \& Priem, R. L. (1995). Consensus-performance research: Theoretical and empirical extensions. Journal of Management Studies, 32(4), 401-417.

Dooley, R. S., Fryxell, G. E. \& Judge, W. Q. (2000). Belaboring the not-so-obvious: Consensus, commitment, and strategy 
implementation speed and success. Journal of Management, 26(6), 1237-1257.

González-Benito, J., Aguinis, H., Boyd, B. K. \& Suárez-González, I. (2010). Coming to consensus on strategic consensus: A mediated moderation model of consensus and performance. Journal of Management, 38(6), 1685-1714.

Hackman, J. R. (1987). The design of work teams. En J. W. Lorsch (Ed.), Handbook of organizational behavior (pp. 315-342). Englewood Cliffs, N. J.: Prentice-Hall.

Homburg, C., Krohmer, H. \& Workman, J. P. (1999). Strategic consensus and performance: The role of strategy type and market-related dynamism. Strategic Management Journal, 20(4), 339-357.

Kellermanns, F. W., Walter, J., Floyd, S. W., Lechner, C. \& Shaw, J. C. (2011). To agree or not to agree? A meta-analytical review of strategic consensus and organizational performance. Journal of Business Research, 64(2), 126-133.

Kellermanns, F. W., Walter, J., Lechner, C., \& Floyd, S. W. (2005). The lack of consensus about strategic consensus: Advancing theory and research. Journal of Management, 31(5), 719-737.

Knight, D., Pearce, C. L., Smith, K. G., Olian, J. D., Sims, H. P., Smith, K. A. \& Flood, P. (1999). Top management team diversity, group process, and strategic consensus. Strategic Management Journal, 20(5), 445-465.

Mahto, R. V. \& Davis, P. S. (2012). Information flow and strategic consensus in organizations. International Journal of Business and Management, $7(17), 1-12$.

Miles, J. \& Shevlin, M. (2001). Applying regression and correlation: A guide for students and researchers. London: Sage Publications.

Mohammed, S. \& Ringseis, E. (2001). Cognitive diversity and consensus in group decision making: The role of inputs, processes, and outcomes. Organizational Behavior and Human Decision Processes, 85(2), 310-335. 
Moreira, T. \& Puente-Palacios, K. (2014). Busca por concordância na tomada de decisóes. En M. M. M. Siquiera (Ed.), Novas medidas de comportamento organizacional (pp. 52-64). Porto Alegre: Artmed.

Pasquali, L. (1999). Testes referentes a construto: teoria e modelo de construção. En L. Pasquali (Ed.), Instrumentos psicológicos: manual prático de elaboração (pp. 37-71). Brasilia: LabPAM/IBAPP.

Puente-Palacios, K. \& Puente, T. (2013). Validación de una Escala de Medición del Consenso Estratégico en Equipos Pedagógicos. Revista de Psicología de la Pontificia Universidad Católica del Perú, 31(2), 227-245.

Ramos-Garza, C. (2009). TMT strategic consensus in Mexican companies. Journal of Business Research, 62(9), 854-860.

Roberto, M. A. (2004). Strategic decision making processes: Beyond the efficiency consensus trade off. Group \& Organization Management, 29(6), 625-658.

Spector, P. E. (1994). Using self-report questionnaires in OB research: A comment on the use of a controversial method. Journal of Organizational Behavior, 15(5), 385-392.

Spector, P. E. (2006). Method variance in organizational research: Truth or urban legend? Organizational Research Methods, 9(2), 221-232.

Tabachnick, B. G. \& Fidell, L. S. (2007). Using multivariate statistics (6th ed.). Needham Heights, MA: Allyn \& Bacon.

Tarakci, M., Ates, N. Y., Porck, J. P., van Knippenberg, D., Groenen, P. J. F. \& Haas, M. (2014). Strategic consensus mapping: A new method for testing and visualizing strategic consensus within and between teams. Strategic Management Journal, 35(7), 1053-1069. Walter, J., Kellermanns, F. W., Floyd, S. W., Veiga, J. F. \& Matherne, C. (2013). Strategic alignment: A missing link in the relationship between strategic consensus and organizational performance. Strategic Organization, 11(3), 304-328.

Recibido: 19 de septiembre, 2014 Aceptado: 30 de octubre, 2014 